\title{
Consensus development conference: treatment of primary breast
}

\section{cancer}

The second King's Fund Forum was held in London from 1 to 3 October 1986. A panel of twelve, half of whom were medical, listened to evidence from experts in public sessions attended by 250 people-including doctors, nurses, and professionals from many disciplines as well as patients and press. After closed sessions the panel discussed its report with the audience and an agreed consensus statement was then presented at a press conference in the form of answers to four questions.

\section{Consensus statement}

One in 12 women in the United Kingdom will develop breast cancer in their lifetime and 25000 will do so every year. It is the commonest cancer in women, accounting for one in five of cancer deaths in women, and is the leading cause of death in women aged 35 to 54 . The clinical course of breast cancer is variable. Some cancers disseminate early, but others may recur only many years later or not at all.

In the past the main treatment for breast cancer was radical mastectomy. This led frequently to side effects such as swelling of the arm and limitation of its movement. Although in recent years there has been a trend towards less radical surgery (used alone or in conjunction with radiotherapy or systemic drug therapy), there is no consensus about optimum treatments.

(1) When a woman is first suspected of having breast cancer, what information is required by the doctor and the patient in order to plan management?

Suspected breast cancer can cause alarm and despondency for the woman and her family. Nine out of ten breast lumps turn out to be benign and it is important that women know this.

If clinical examination suggests that cancer is possible, the general practitioner needs to be sensitive to the woman's fears and expectations and to the extent to which she wishes to be involved in decisions about her care. Some patients want to consider all the options, while others do not. She should be referred promptly for diagnosis. The general practitioner has a responsibility to outline what the patient should expect when she reaches hospital and explore with her how she will share her concern with those close to her.

At the hospital all new patients should be seen and examined by the consultant at their first visit. All staff should be aware of the anxiety women face and should arrange that waiting times are minimal and conditions are comfortable. Mammography may give additional information and define abnormalities in either breast. If either clinical examination or mammography reinforces the suspicion of cancer a sample from the breast must be obtained for microscopic examination.

This may be done using fine needle aspiration cytology or Trucut needle biopsy under local anaesthesia. In a minority of women these

The panel comprised: Dame Mary Donaldson (Chairman), Professor John Bain, Professor David Carter, Dr Iain Chalmers, Dr Jocelyn Chamberlain, Ms Christine Hancock, Professor Alan Maynard, Rabbi Julia Neuberger, Dr Ann Oakley, Dr Chris Paine, Mr Nick Ross, Professor Michael Whitehouse.

Invited experts presenting evidence were: Professor M Baum, Professor R Blamey, Dr D Brinkley, Mr M Buxton, Ms S Denton, Ms C Faulder, Professo Sir Patrick Forrest, Dr S Gore, Mr J Hayward, Professor L Hughes, Dr P MacGuire, Dr R Peto, Dr M Pike, Professor R Sellwood, Dr I Smith, Dr D Wild. investigations will not yield adequate samples, and open surgical biopsy may be required. This should be a separate diagnostic procedure so that there is an opportunity for the woman to know the results and to discuss and decide treatment options with the surgeon. Frozen section biopsy followed immediately by mastectomy is rarely justified.

Once breast cancer is confirmed, factors which may influence the choice of treatment include the patient's age, menopausal state, tumour size and local extension, spread to the regional lymph nodes, and presence of distant metastases. Spread to the nodes cannot be determined accurately by clinical examination, and axillary nodes should be sampled at the time of breast surgery. Spread to these nodes by cancer usually indicates systemic disease. Radiographs of the chest and sometimes of the lumbar spine and pelvis will be undertaken, but isotope scanning is not usually necessary.

Prognostic indices which bring together consideration of tumour size, histological grade, oestrogen receptor state, and more extensive node sampling discriminate more accurately between good and poor prognosis patients. The value of this approach in deciding treatment remains unclear.

The patient must be offered every opportunity of full discussion of the implications of these results. A woman counsellor may help the patient to understand and adjust to her diagnosis and treatment options.

(2a) For various subgroups of patients what are the best forms of initial local treatment (surgery, radiotherapy) in terms of local recurrence, distant spread, long term survival, and quality of life, and how do these influence the need for other therapy?

The effects of all procedures on survival and recurrence rates, and on the quality of life of the woman, require careful evaluation. Such evaluation should use a wide range of quality of life measures.

There is no evidence that mastectomy or more extensive surgery, as opposed to local removal of the tumour, leads to longer survival. The risk of local recurrence is greater with breast conservation. However, this risk can be reduced substantially by radiotherapy, although there is no evidence that radiotherapy prolongs life. The treatment takes several weeks and has limited term side effects both locally and on the patient as a whole. Travelling distance will also be an important factor for some patients. The long term effects of radiotherapy still require careful study.

The possibility of reconstructive surgery should be discussed with all women in whom a significant loss of breast tissue will be necessary. Reconstruction may take place at the time of the original operation, in a unit with requisite skills, or may take place later. All patients undergoing mastectomy without reconstruction should be given advice about a prosthesis by the surgeon and a woman member of staff experienced in the selection and fitting of breast prostheses.

For tumours which are multifocal or affect a large portion of the breast mastectomy will often be the best surgical treatment. Mastectomy may also be preferred by some women with smaller tumours to reduce the risks of local recurrence and the need for adjuvant radiotherapy.

Gross spread to the axilla is normally treated by surgical axillary clearance, and radiotherapy is reserved for recurrence. However, these patients will usually have large primary tumours, so that modified radical mastectomy (without radiotherapy) may be preferable. Women with locally advanced cancer affecting the skin or underlying muscle, and those found to have metastatic disease, will generally benefit from radiotherapy, endocrine therapy, and/or chemotherapy. This complicated issue is not considered here. 
(2b) For various subgroups of patients what are the best forms of systemic treatment (chemotherapy, endocrine therapy) in terms of local recurrence, distant spread, long term survival, and quality of life?

An overview of all randomised trials shows that relapse rates can be reduced in women under 50 with cytotoxic drugs immediately after initial surgical treatment. Single agents have not been shown to reduce mortality rates at five years. Use of a combination of agents (cyclophosphamide, methotrexate, and 5-fluorouracil-CMF) in women with affected nodes reduces their risk of death over the subsequent five years from $36 \%$ to $27 \%$ compared with similar women who had either single agent or no chemotherapy. Any benefits are substantially less in women over 50 . Furthermore, these drugs may have unpleasant side effects, so their costs and benefits must be carefully assessed. There is no evidence that courses of treatment lasting more than six months enhance this effect. Indeed a study with a 20 year follow up shows a reduced mortality rate after a six day course of cyclophosphamide.

Data from all randomised trials assessing the effects of destroying ovarian function (by surgical removal or irradiation) show reductions in mortality in women under 50 comparable to those achieved with CMF. The beneficial effect of CMF in women under 50 may be partly due to its effect on ovarian function. Destroying ovarian function results in menopausal symptoms and an increased incidence of cardiovascular disease.

Endocrine therapy with tamoxifen given for two years after initial treatment in patients over 50 results in both a reduced relapse rate and a reduction in death from $30 \%$ to $24 \%$ over five years. In patients under 50 there is so far no convincing evidence of a reduction in mortality after treatment with tamoxifen, although there is some evidence of a reduced relapse rate. Tamoxifen has minimal side effects compared with CMF, but its long term effects are unknown.

(3) What are the pros and cons of different degrees of participation by women in deciding about their own treatment?

Although some women do not wish to take part in decisions about treatment, others feel excluded and resentful if they are not fully informed and consulted. In general, doctors underestimate the amount of information patients want.

While some women feel threatened by being given unsolicited information, and their confidence in treatment may be undermined if the doctor seems uncertain, there are strong arguments in favour of women's participation in treatment decisions. These are that: if the woman is fully involved in decisions about her own care without feeling patronised she is more likely to feel positive about the treatment she elects, however distasteful it may be; if she is free to refuse treatment, frank discussion of her reasons for refusal will minimise resentment on either side and a relationship of trust will be established, making it easier for both parties should problems occur. Openness also makes it easier for the woman to understand the need for a randomised trial of alternative treatments and why she is being asked to participate.

The woman needs time to take in the news that she has breast cancer. Because immediate treatment is not essential a woman can safely be offered an interval before treatment decisions are made. She should be told that she is welcome to bring a family member or friend with her at the next consultation. Again it is essential that counselling should be available, supplemented by a booklet or tape recording which may be taken home.

(4) How should services for treating breast cancer be organised to maximise benefits and minimise disadvantages?

As yet there is no evidence that the outcome of treatment in terms of survival or recurrence is any better in specialist units than general hospitals. None the less, a strong case can be made for grouping together the services for women with breast cancer. Surgeons with no special interest in breast cancer are less likely to be aware of trial results and other advances. They may also be less skilled in appreciating the woman's need for information and psychological and practical support.

In each health district one surgeon should be encouraged to take primary responsibility for running and auditing a service for women with breast cancer. This will involve the establishment of an outpatient breast clinic incorporating the services of a trained nurse counsellor. The clinic needs to be backed up by mammography using dedicated equipment and staffed by an experienced radiographer and radiologist. A histopathologist with experience of breast cytology is also required.

The team of surgeon, radiologist, pathologist, and nurse will also need to consult closely with a radiotherapist and/or oncologist, preferably in a joint clinic. These links should help to minimise travel to radiotherapy centres.

After treatment has been started the breast team, together with the general practitioner, needs to be aware of the likelihood of practical problems, as well as of depression or anxiety, which can be successfully treated. In each district there should be a psychiatrist attached to the breast team. Good communication between the general practitioner and the breast team will ensure that both parties are aware of the services provided, both in hospital and in the community, and of the woman's adjustment to knowledge of her disease and its treatment. Self help support groups in the community are useful in assisting with both practical problems and psychological support, provided that there is appropriate training.

These services do require some additional resources as well as reorganisation, together with training of certain categories of staff. The present state of knowledge of both the costs of care and its outcome in terms of quality adjusted life years does not permit any assessment of the value of different patterns of care, nor of how they compare with the value of other health procedures.

These remarks apply to existing services. If a screening programme were to be introduced the resources for diagnostic services would need an enormous expansion. The breast clinics suggested would be a useful starting point from which to develop a screening programme.

Much of the evidence on which the panel's recommendations are based comes from randomised trials in which women with breast cancer have participated when the best treatment has been unknown. Advances in knowledge are likely to continue to come from properly controlled trials of different treatments. Women should not be entered into trials without the opportunity to give their informed consent. In such trials information should be collected not only on survival and recurrence but also on quality of life, on costs both to the health service and the woman, and on women's satisfaction with their care.

A woman of 72 has developed considerable kyphosis over the past few years. The condition appears to be familial, affecting her mother's family on the female side, and is associated with osteoporosis. Her daughter (aged 40) is worried that she too may develop the condition. What advice could she be given to limit the chances of a deformity developing?

It does not necessarily follow that the daughter will be similarly affected. Several factors are likely to influence what happens. First and foremost there is the question of the onset of her menopause. If the daughter is already menopausal her chances are increased as there will be a longer time for the effect of oestrogen loss to affect her skeleton. If she has led an active and vigorous life and, in her early years, amassed a good skeletal store of calcium the chances of her developing kyphosis are reduced. In addition, if she is obese she will have less risk of becoming osteoporotic as in a woman there is a definite correlation between leaness and poor musculature and osteoporosis. The best way for her to reduce the risk of osteoporosis will be to exercise, and when she does become menopausal-if not already so- to take a combination of oestrogen and a progestogen with calcium supplement to maintain her skeletal strength.- - LESLIE KLENERMAN, consultant orthopaedic surgeon, London. 